\title{
Control of Wall Band Splitting in Streptococcus faecalis
}

\author{
By ARTHUR L. KOCH ${ }^{1}$ AND MICHAEL L. HIGGINS 2* \\ ${ }^{1}$ Program on Microbiology, Department of Biology, Indiana University, Bloomington, \\ Indiana 47405, USA \\ ${ }^{2}$ Department of Microbiology and Immunology, Temple University School of Medicine, Philadelphia, \\ Pennsylvania 19140, USA
}

(Receined 6 October 1983)

Computer reconstructions of 659 and 1325 whole mounted, shadowed cells, randomly chosen from cultures of Streptococcus faecalis undergoing balanced growth and doubling in mass every $83 \mathrm{~min}$ and $30 \mathrm{~min}$, respectively, were used to analyse the cell cycle. The size limits and duration of phases of the cell cycle were estimated by applying a method previously described by the authors, details of which are given here to allow others to use the method. Deeply constricted cells whose primary septal radius, $R_{\mathrm{s}}$, was less than or equal to $0.18 \mu \mathrm{m}$ were considered as belonging to an $E$-phase ending the cell cycle. The statistical parameters of these $E$-phase cells were used to calculate the mean and coefficient of variation of dividing cells. These latter values, in turn, predicted the moments of the total population well enough so that the method's assumptions were judged adequately satisfied. Therefore, the method was considered applicable to other phases and sub-phases of the cell cycle of these two cultures. The $E$-phase cells were further classified as having either 0,1 or 2 secondary growth zones, allowing us to calculate the percentage of newborn cells without growth zones. In the slow-growing cells, $69 \%$ of the cells arose with no growth zone. On the other hand, in more rapidly growing cells $16 \%$ of the cells or less arose with no growth zone. Our calculations showed that they could exist without a growth zone for only 2 and $0 \cdot 1 \mathrm{~min}$, respectively. We also classified cells as possessing a 'birth site' if the volume between the two daughter bands was greater than 0 , but less than $0.06 \mu \mathrm{m}^{3}$. From the statistical properties of such cells with new growth zones, the mean pole time, $W$, was estimated. We also estimated $W$ from the size of cells in $E$-phase. The major conclusion is that the pole time is only slightly greater than the mass doubling time at both growth rates. Since DNA synthesis in $S$. faecalis takes longer ( $C=50$ to $52 \mathrm{~min}$ ) than the mass doubling time in rich medium ( $30 \mathrm{~min}$ ), a new round of chromosome replication must be initiated before the old round of synthesis is completed (dichotomous replication). Consequently, wall band splitting and initiation of chromosome replication do not occur simultaneously. It was also concluded that the cell initiates wall band splitting, resulting in pole formation and cell division, when the growth zones cannot function rapidly enough to allow the increase of surface area required to accommodate continuing production of cytoplasm.

\section{INTRODUCTION}

Streptococci have the simplest cell growth pattern of any free-living cell. The wall of the organism grows in only one way (Higgins \& Shockman, 1976): a new site forms by the production of a nascent septum under a wall band. As the nascent septum is assembled, a division furrow appears which splits the wall band overlying the septum, creating two new bands. The furrow splits the septum allowing it to separate and expand into two layers of peripheral wall. The septum grows into the cytoplasm by the addition of peptidoglycan to the invading edge of the septum. Additional peptidoglycan seems to be required to adjust the thickness of the septum as it splits and expands into peripheral wall. The inner surface of the septum extends progressively until the septum closes. Shortly thereafter the division furrow constricts until the septum 

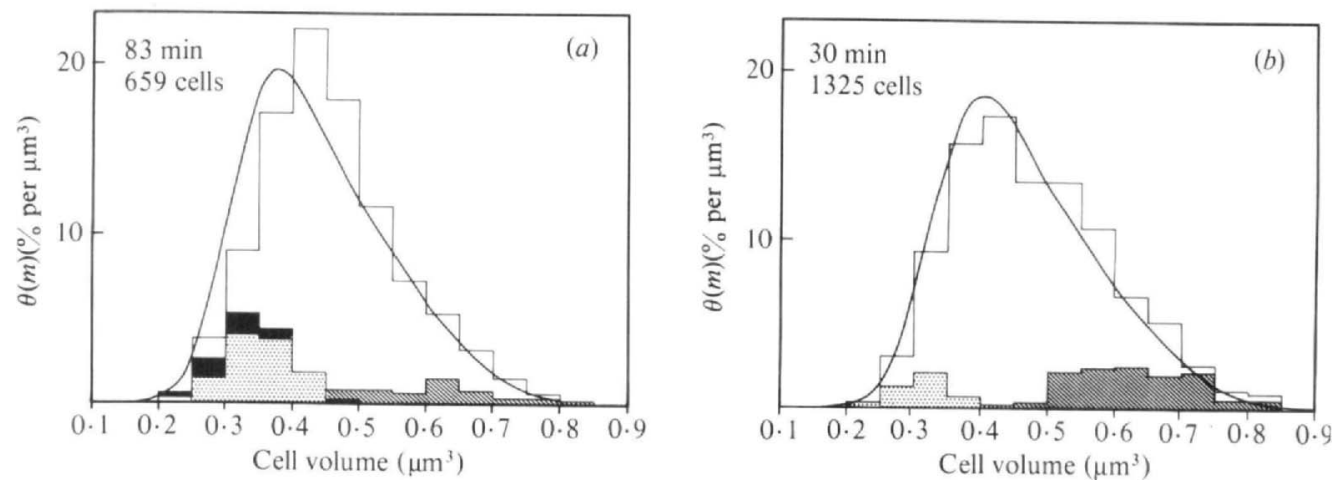

Fig. 1. Distribution of volumes of cells from various phases of the cell cycle. The total cell population is shown by the unfilled histogram. The population of cells in the $E$-phase, which have almost divided, is shown with slanted hatching. The stippled histogram represents cells with primary 'birth sites' (see text). The solid area shown in $(a)$ corresponds to 'unit cells'. For clarity they have been superimposed on the stippled distribution. The mass doubling time and the total number of cell replicas taken from balanced culture are shown on the graphs. The continuous curve is the distribution of the total population predicted from the data concerning the $E$-phase population alone. $\theta(m)=$ distribution of cell sizes.

has been completely severed and two finished poles result. Since wall bands can split, creating secondary growth zones before the primary growth zones have completed pole formation, this process is reminiscent of dichotomous replication of chromosomes observed in both Gram-positive and Gram-negative bacteria. However, the quantitative analysis presented here shows that the timing of splitting of the wall bands and the rate of pole development at different growth rates are quite different from the timing of initiation and rate of chromosome replication.

Information about the phases of the cell cycle is inherent in the distributions of cell size, measured on samples taken from balanced steady state cultures. The timing of cell cycle events in Streptococcus faecalis was deduced from the analysis of three-dimensional reconstructions of electron micrographs of replicas of whole cells taken from exponentially growing cultures. These reconstructions were used to calculate the frequency distributions of the cell volumes (Fig. 1) of the entire population and subpopulations such as those involved in the terminal stages of division (end- or $E$-phase) or those that have no growth zones, etc.

\section{ANALYSIS OF THE CELL CYCLE}

Heretofore there have been no good techniques to estimate initiation and finishing sizes and ages of cell cycle phases. There have been only approximate methods based on the questionable approximation that all cell cycles have the same duration. However, we have recently developed a method (Koch \& Higgins, 1982) so that these sizes and ages can be computed, provided that cells grow and divide according to assumed rules. Computer simulation is then used to build theoretical distributions of cell volumes for a population based on these rules for a range of the parameters. From the graphs and formulae given by Koch \& Higgins (1982) it is possible to estimate the average time when cells start and finish cell cycle phases, from the average sizes of cells when a phase is initiated and when it is finished. If it can be determined by internal consistency that the simulated distribution fits the actual measurements of $E$-phase cells and the total population of cells, then the computer results of simulations of the distributions of other cell cycle phases can be used to give an estimate of average size and ages of cells when they start and finish these phases.

To understand how this procedure works, consider a much more restrictive and idealized set of assumptions than is applicable to actual bacterial cultures. In this illustrative case, all cells grow in an identical manner. Thus all cells would be born at an invariant size, grow exponentially to an invariant division size twice that of the birth size and then divide exactly in half to form equal-sized daughters. In an asynchronous population, distribution of the ages of cells 

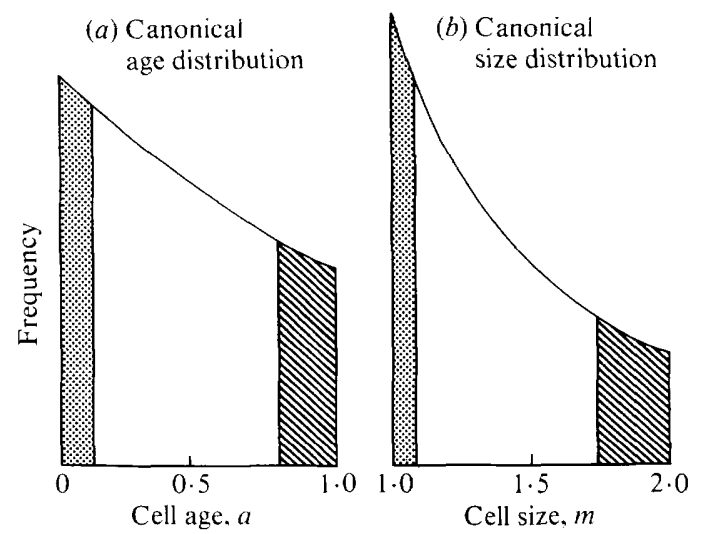

Fig. 2. Distribution of ages and sizes for an idealized binary fission cell cycle. $(a)$ The 'canonical' age distribution; (b) the "canonical' distribution of sizes (see Koch \& Schaechter, 1962). Also shown are the $E$-phase (hatched) and the $S$-phase (stippled), comprising $15 \%$ of the total cells in the growing population. The durations of the phases can be calculated from equations (1) and (2). Alternatively they can be calculated from the observed properties of the phases from:

$$
T_{x}=-T \log _{2}\left(1-H\left(1+q_{e}^{2}\right) \bar{e}^{2} / 4\right)
$$

and

$$
T_{x}{ }^{\prime}=T \log _{2}\left(1+\Sigma\left(1+q_{s}^{2}\right) \bar{s}^{2} / 2\right)
$$

where $H, \vec{e}$, and $q_{e}$ and $\Sigma, \bar{s}$, and $q_{s}$ are the frequency, mean size, and coefficient of variation of the $E$ - or $S$-phases.

is the well-known 'canonical' distribution (Fig. 2a), so called because it is the idealized case of zero variation of age at division. The corresponding 'canonical' distribution of cell volumes for such a perfect cell cycle (Koch \& Blumberg, 1976) is shown in Fig. 2(b). The distributions of cell volumes obtained from actual cells are quite different (compare Fig. 1). As shown by Koch \& Schaechter (1962) the major reason is due to the variability in the sizes of cells at division, which results in an even greater variability in the age of division.

For the moment, consider two phases: one that starts with the beginning of the cycle (an $S$ phase) and another that ends the cycle (an $E$-phase). For illustrative purposes in Fig. 2, we have assumed that both of these contain $15 \%$ of the cells of a culture in balanced growth. The duration of the $E$-phase can be calculated from

$$
T_{x}=T \log _{2}(1+Y)=0 \cdot 2016
$$

and for the $S$-phase from

$$
T_{x}^{\prime}=T\left[1-\log _{2}\left(2-Y^{\prime}\right)\right]=0 \cdot 1125
$$

which are modifications of equations given by Mitchison (1971) and used by Gibson et al. (1983). $Y$ and $Y^{\prime}$ are the frequency of the $E$ - and $S$-phase cells [in the Koch \& Higgins (1982) nomenclature they are designated as $H$ and $\Sigma]$. At the right hand side of these equations, the duration of the $E$ - and $S$-phase are expressed as fractions of the mass doubling time. The same fractional times could be calculated from data presented in the format of either Fig. 2(a) or Fig. $2(b)$ because, of course, the frequencies are the same either way. But for studies of bacteria, the need for high resolution for the accurate estimation of size precludes the following of individual cells during their growth by means of phase-contrast microscopy to construct an age distribution, and only the distributions as a function of cell size can be obtained from electron microscope studies.

Equations (1) and (2) only strictly apply if the $E$-phase, the $S$-phase and the total phase have the same duration for all cells, and that a random population is sampled from a culture undergoing strict balanced growth. The new, more general alternative procedure involves additional 
types of data beyond the frequencies: these are the mean sizes (volumes) of the cells in the $E$-or $S$-phases and the coefficient of variation of these sizes. The mean $(\bar{e}$ or $\bar{s})$ and variation of cell volumes of a phase ( $q_{e}$ or $q_{s}$ ) are experimentally measurable from data expressed as size distributions. The more elaborate equations needed for the canonical case are given in the legend to Fig. 2 ; this provides a second way to translate the percentage incidence of a phase ( $H$ or $\Sigma$ ) into the duration of the $E$ - or $S$-phase which can be extended to cases when the division size varies.

For cases where there is variation, the computer modelling permits us to calculate (actually extrapolate) the mean size and coefficient of variation of dividing cells. This is an extrapolation since, had we chosen a more restricted $E$ - or $S$-phase which included fewer cells (corresponding to only the very last or very first stages of the cell cycle), then the actual measurements would reflect the size properties of 'dividing cells' or 'newborn cells'. From the mean and coefficient of variation of dividing cells so estimated, it is possible to calculate the size distribution of the total population (Koch \& Schaechter, 1962; Koch, 1966, 1977; Koch \& Blumberg, 1976; Koch \& Higgins, 1982). The computer programs do this quite simply by averaging the canonical distributions for cells with a range of birth and division sizes. Examples of this are shown in Figs $1(a)$ and $1(b)$ by the smooth curves; these are the population distribution of all cells based on the frequency distribution of $E$-phase cells alone.

The crux of the new method is that if the computer-simulated distribution agrees with the actual size distribution derived from a large population of cells sampled from a culture in balanced growth, it is reasonable to assume that the growth rules upon which the program was constructed are sufficiently valid to be used to examine further other phases or stages of the cell cycle. In the present application to cultures of $S$. faecalis, the calculated distributions (the continuous line in Figs $1 a$ and $1 b$ ) agree quite well with the actual measurements. Therefore, the same program could be used to calculate the distributions of other phases and subpopulations of these cultures.

\section{Steps of the Koch \& Higgins method}

Application of the method requires reading values from graphs or using the polynomial regressions presented by Koch \& Higgins (1982). The latter process has been simplified by incorporating the regressions into a programmable pocket computer program. The method consists of seven steps; the first six steps are needed to justify the method, and the seventh step applies it to a cell phase of interest. The steps are:

(1) To measure the volume of a sufficiently large number of cells from a population in a welldefined state of balanced growth to define accurately the population size distribution. Our two populations had 659 and 1325 cells after a few abnormal cells were removed (see below).

(2) To compute the first four moments of the distribution in order to calculate the mean, coefficient of variation, skewness, and kurtosis. These are shown under the heading 'IV. Observed for total population' in Table 1.

(3) To measure the size distribution of that part of the population that is in a phase of the cell cycle that ends with cell division, then to calculate the mean, $\bar{e}$; the coefficient of variation, $q_{e}$; and frequency of the phase in the total population, $H$. In the present application, we arbitrarily chose the criterion that $R_{\mathrm{s}}$ should be less than $0 \cdot 18 \mu \mathrm{m}$ to identify cells as being in the $E$-phase. Whilst this is a completely arbitrary criterion, we can be sure that all cells in the population must pass through this phase which ends with cell division.

(4) To calculate the mean finishing size, $e_{f}$, and coefficient of variation of the size at division $q$, from $\bar{e}, q_{e}$, and $H$. In this application it was done with a Hewlett-Packard HP41C program which codes the formulae given by Koch \& Higgins (1982). These results are shown under 'II. Calculated for dividing cells' in Table 1.

(5) To calculate the expected values of the parameters of the population of total cells from $e_{f}$ and $q$. This also was done on the HP41C from the formulae previously given. These results are shown in Table 1 under the heading 'III. Predicted for total population'.

(6) To compare the expected statistical moments of total cell population (III) with the observed values (IV). In the present case, agreement of the mean and coefficient of variation is good. Consequently, the assumptions (of balanced growth, of independent sizes at successive 
Table 1. Cell cycle parameters for Streptococcus faecalis

See text for details. CV, coefficient of variation; $\gamma_{2}$, kurtosis; $\beta_{1}$, skewness; 'secondary', secondary growth zone.

I. Observed for $E$-phase cells:

II. Calculated for dividing cells:

III. Predicted for total population:

IV. Observed for total population:

V. Mean birth volume, $s_{i}$

$1 / 2$ of $e_{f}$

Unit cells

Primary birth sites

VI. Mean primary volume of $E$-phase sub-categories

No secondary

1 secondary

2 secondaries

VII. Mean pole time, $\bar{W}$

0 secondaries $T\left[1+0.5 \log _{2}\left(e_{f} / \bar{e}_{i_{0}}\right)\right]$

1 secondary $T\left[1+0 \cdot 25 \log _{2}\left(e_{f} / \bar{e}_{i_{1}}\right)\right]$

2 secondaries $T\left[1+\log _{2}\left(e_{f} / e_{i_{2}}\right)\right]$

Average

Corrected for unit cells

\begin{tabular}{|c|c|c|c|c|}
\hline \multirow{2}{*}{$\begin{array}{l}\text { Doubling } \\
\text { time, } T \ldots\end{array}$} & \multicolumn{2}{|c|}{$83 \mathrm{~min}$} & \multicolumn{2}{|c|}{$30 \mathrm{~min}$} \\
\hline & $\begin{array}{l}\text { Volume } \\
\left(\mu \mathrm{m}^{3}\right)\end{array}$ & $\begin{array}{l}\text { No. of } \\
\text { cells }\end{array}$ & $\begin{array}{c}\text { Volume } \\
\left(\mu \mathrm{m}^{3}\right)\end{array}$ & $\begin{array}{l}\text { No. of } \\
\text { cells }\end{array}$ \\
\hline $\begin{array}{l}\text { mean } \bar{e} \\
\text { CV } q_{e} \\
\text { freq. } H \\
\text { mean } e_{f} \\
\text { CV } q \\
\text { mean } \bar{t} \\
\qquad \begin{array}{l}q_{t} \\
\beta_{1} \\
\gamma_{2} \\
\bar{t} \\
q_{t} \\
\beta_{1} \\
\gamma_{2}\end{array}\end{array}$ & $\begin{array}{l}0.6154 \\
0 \cdot 1498 \\
0.0516 \\
0.6423 \\
0 \cdot 1492 \\
0.4402 \\
0.2495 \\
0 \cdot 6045 \\
3 \cdot 0214 \\
0 \cdot 4588 \\
0 \cdot 2268 \\
0 \cdot 6424 \\
3 \cdot 3550\end{array}$ & $659^{*}$ & $\begin{array}{l}0.6424 \\
0 \cdot 1504 \\
0.0792 \\
0 \cdot 6789 \\
0 \cdot 1489 \\
0.4653 \\
0 \cdot 2493 \\
0.6044 \\
3 \cdot 0199 \\
0 \cdot 4807 \\
0 \cdot 2447 \\
0.5035 \\
2.7407\end{array}$ & $1325^{*}$ \\
\hline & $\begin{array}{l}0.3212 \\
0 \cdot 3054 \\
0.3343\end{array}$ & $\begin{array}{l}34 \\
21 \\
88\end{array}$ & 0.3395 & $\begin{array}{r}105 \\
1 \\
61\end{array}$ \\
\hline $\begin{array}{l}e_{i_{0}} \\
e_{i_{1}} \\
e_{i_{2}}\end{array}$ & $\begin{array}{l}0.6119 \\
0.6315 \\
0.5461\end{array}$ & $\begin{array}{r}21 \\
5 \\
8\end{array}$ & $\begin{array}{l}0.6326 \\
0.5572 \\
0.5601\end{array}$ & $\begin{array}{r}9 \\
16 \\
80\end{array}$ \\
\hline & $\begin{array}{l}\text { Time } \\
(\min )\end{array}$ & $\begin{array}{l}\text { No. of } \\
\text { cells }\end{array}$ & $\begin{array}{l}\text { Time } \\
(\min )\end{array}$ & $\begin{array}{c}\text { No. of } \\
\text { cells }\end{array}$ \\
\hline
\end{tabular}

$\begin{array}{rrrr}85 \cdot 9 & 21 & 31 \cdot 5 & 9 \\ 83 \cdot 3 & 5 & 32 \cdot 1 & 16 \\ 102 \cdot 4 & 8 & 38 \cdot 3 & 80 \\ 89 \cdot 4 & 34 & 36 \cdot 8 & 105 \\ 88 \cdot 0 & & 36 \cdot 8 & \end{array}$

\footnotetext{
* The total population contains 662 and 1333 cells, respectively. A few cells were eliminated from the calculations (see text for details).
}

divisions, of a Gaussian division distribution, of exponential growth of individual cells, of the division of the mother cell into equal-sized daughters, and that the duration of the $E$-phase is the same for all cells within each population) are a satisfactory representation of the cell populations at both growth rates. Therefore, we proceeded to step 7 .

(7) To apply to phases of interest the appropriate regressions and graphs given by Koch \& Higgins (1982).

\section{METHODS}

Cell growth. Cells of S. faecalis ATCC 9790 were grown as described previously (Hinks et al., 1978a). Tryptophan concentration was reduced to $20 \mu \mathrm{g} \mathrm{ml}^{-1}$ to eliminate chaining. The growth rate of each culture was regulated by eliminating glutamine from the chemically defined medium, and providing the glutamate at 20 and $300 \mu \mathrm{g} \mathrm{ml}^{-1}$ for the 83 and $30 \mathrm{~min}$ cultures. Before being fixed for electron microscopy, cells were allowed to go through six exponential-phase mass doublings at the growth rate being studied. Growth was monitored turbidimetrically at $675 \mathrm{~nm}$ in a Spectronic 20 spectrophotometer (Bausch and Lomb, Rochester, NY, USA). Optical density readings were adjusted to agree with Beer's Law and expressed as adjusted optical density (AOD).

Electron microscopy. Sample preparation and quantification of electron micrographs were described previously (Edelstein et al., 1980). Photography was done with a Siemens Elmiskop 1-A electron microscope (Siemens Corp., West Berlin) at an instrumental magnification of $6000 \times$. The dimensions and volume of the cells and their parts 
were stored in a computer in such a way that they could be sorted at will. Two classifications were particularly important : cells containing a primary growth zone whose septal radius was less than $0 \cdot 18 \mu \mathrm{m}$, and cells with 'birth sites'. The former included the $5-8 \%$ of the cells that constitute a phase that ends with cell division. The other classification depended on the cellular volume included between two sister wall bands. We have arbitrarily considered those cells with this volume greater than zero but less than $0.06 \mu \mathrm{m}^{3}$ as having 'birth sites'. Birth sites were exhibited by cells with one, two or three growth zones.

\section{RESULTS}

\section{Construction of distributions}

Figure 1 shows the distribution of sizes of the populations of cells taken from two balanced, growing cultures with mass doubling times of $83 \mathrm{~min}$ and $30 \mathrm{~min}$. Superimposed on these histograms are histograms of cells in various categories. Some categories constitute obligatory cell phases while others do not. The $E$-phase cells are shown with slanted hatching. We also consider a group of cells with a primary birth site with a growth zone with volumes $\leqslant 0.060 \mu \mathrm{m}^{3}$. Neither cells with primary birth sites nor cells with secondary birth sites constitute an obligatory phase, since not all cells initiate wall band splitting in the same relationship to cell division. But a priori cells with only a primary birth site must represent cells very shortly after cell division, because although the volume of the cells seems to increase continuously, a cell can expand a completed pole by only a small amount. In addition to $E$-phase and birth site categories, 21 of the 662 cells of the $83 \mathrm{~min}$ population and 1 of the $133330 \mathrm{~min}$ population had neither a primary nor a secondary growth zone. The properties of these cells, previously designated 'unit cells' (Edelstein et al., 1980), were also considered in the analysis of the cell cycle.

In carrying out the analysis of these populations, a decision was subjectively made to reject a few of the very large cells from further consideration. It has been pointed out (Koch, 1980) that there are a few abnormal cells in many of the bacterial populations that have been carefully examined. These abnormal cells grow and divide much more slowly than expected from the behaviour of the bulk of cells in the same culture. This causes little effect unless the tails of the population size distribution are of interest. Of course, a few cells that fail to divide, but continue to grow larger at a decreased rate, markedly change the shape of the distribution and greatly affect the higher moments of the size distribution. Since the properties of the total population and $E$-phase size distributions are essential to our analysis, it was necessary to remove such 'pathological' cells. If they were retained and classified as $E$-phase cells, a significant overestimation of the size of dividing cells would be made, and if they were classified as non $E$-phase cells the statistical parameters of the total population would be distorted. The choice of how many cells were to be removed was made by successively removing the largest cell from the total distribution and at each step calculating the effect of the removal of the moments of the 'observed for total population' (step 2) as well as on the 'predicted for the total population' computed from the $E$-phase data (steps 4 and 5).

As the largest cell was successively removed from the distributions, the parameters of the observed size distributions from step (2) and those calculated from the $E$-phase cells (step 5) approached each other and then diverged in opposite directions. In choosing how many cells to eliminate, we placed most emphasis on the coefficient of variation of the total population, but the mean, skewness, and kurtosis statistics were also considered. Excluding 8 cells out of 1333 $30 \mathrm{~min}$ cells, and 3 cells out of $66283 \mathrm{~min}$ cells, gave the optimum agreement of statistical parameters; these are shown in Table 1, sections III and IV.

\section{Properties of E-phase cells}

Under the definition we have chosen for the $E$-phase, the frequency of nearly-divided cells in the $83 \mathrm{~min}$ population, $H$, was $34 / 659=0.0516$ and in the $30 \mathrm{~min}$ population was $105 / 1325=0.0792$. These frequencies are small and, therefore, this phase represents only a brief part of the cell cycle. The actual durations were calculated from these frequencies and the mean values and coefficient of variation of the volumes of $E$-phase cells (data given in Table 1). First, the $e_{i}$ and $e_{f}$ values were calculated from equations (3, 4, and 5) of Koch \& Higgins (1982). The durations were then computed assuming exponential growth from $T \log _{2}\left(e_{f} / e_{i}\right)$. The $e_{f}$ values 
are given in Table 1 ; the $e_{i}$ values were 0.6118 and $0.6308 \mu \mathrm{m}^{3}$. Thus, for the two populations, the duration of the $E$-phase was $83 \log _{2}(0.6423 / 0.6118)=5.8 \mathrm{~min}$, and $30 \log _{2}(0.6789 /$ $0.6308)=3.2 \mathrm{~min}$. Although the $E$-phase has been defined arbitrarily here, it nevertheless provides a useful and valid way for calculating quantities of importance about the cell cycle that are not arbitrary, such as the mean division size, $e_{f}$, and its coefficient of variation, $q$.

\section{Properties of nascent cells}

A picture of the cells newly arising from division was formed by extrapolating from $E$-phase cells, by examining unit cells, and by considering cells with only primary birth sites. The major item of interest was the proportion of cells not having growth zones at the instant that they arose by division. This frequency was estimated from the proportion of cells with two, one, or zero secondary growth zones among the $E$-phase cells. From the numbers of $E$-cells in these various sub-categories (see bottom part of Table 1), the expected frequencies of unit cells among newlyarisen cells was:

$$
\frac{1 \times 5+2 \times 21}{2 \times 8+2 \times 5+2 \times 21}=0.69 \text { and } \frac{1 \times 16+2 \times 9}{2 \times 80+2 \times 16+2 \times 9}=0.16
$$

for the $83 \mathrm{~min}$ and $30 \mathrm{~min}$ populations. However, these values are slight overestimates because the $E$-phase, although brief, is not infinitely short (the calculation would have been more exact if we had chosen $R_{\mathrm{s}}$ much closer to zero, but then we would have had to examine a much larger population to find enough cells in the phase for meaningful statistics).

Comparison of the $69 \%$ of the cells calculated to arise as unit cells with the $21 / 659=3 \cdot 19 \%$ of cells in the $83 \mathrm{~min}$ population which were observed to be unit cells led to an estimate of the mean length of time that a newly arisen unit cell will exist before its wall band splits. This was done by the procedure of Koch \& Higgins (1982), but with little difference in results compared to the use of equation (2). For the latter approximate method, $Y^{\prime}$ was set at $21 / 659 \times 0.69=0.0462$ and yielded a value of $T_{x}{ }^{\prime}$ of $2.8 \mathrm{~min}$. For the former method, the average duration that cells remain as unit cells was calculated from $T \log _{2}\left(s_{f} / s_{i}\right)$. The $s_{f}$ and $s_{i}$ values were obtained from the data inserted in equations (6), (7) and (8) of Koch \& Higgins (1982). From $s_{i}$ of 0.3054 and $s_{f}$ of $0.3105 \mu \mathrm{m}^{3}$ and $T_{2}$ the $83 \mathrm{~min}$ cells, $T_{x}{ }^{\prime}$ was found to be only $2.0 \mathrm{~min}$.

Comparison of the $16 \%$ of cells calculated to arise as unit cells in the $30 \mathrm{~min}$ population with the $1 / 325=0.075 \%$ observed as unit cells showed that unit cells quickly form a new growth zone. Setting $Y^{\prime}$ to $1 / 1325 \times 0 \cdot 16=0.0047$, we obtain an estimate of $T_{x}{ }^{\prime}$ of only $0 \cdot 1 \mathrm{~min} . T_{x}{ }^{\prime}$ was expected to be much smaller for the more rapidly growing culture, in part because the entire cell cycle appears to speed up, and in part because hydrostatic pressure would increase extremely rapidly in a cell with no growth zone.

The newly-arisen cells had a different mean size depending on whether they had a functioning growth zone as they arose. The mean birth cell volume (Table 1 , section V) was estimated in several ways. The first approach was by halving the mean final size for the phase ending the cell cycle, $e_{f}$, taken from Table 1 , section II. This, of course, averaged cells both with and without growth zones. The second approach used the volumes of cells with no primary or secondary growth zone ('unit cells') (for statistical reasons, this can only be done for the 83 min population). Since this class of cells does not constitute a phase, the methods of Koch \& Higgins (1982) do not strictly apply. However, it could be demonstrated by more elaborate calculations that the error is small and we assumed that the fraction of the population in the phase was the observed 21/659. From equations (6) and (8) given in Koch \& Higgins (1982) and from the observed mean volume of the unit cells of $0.3079 \mu \mathrm{m}^{3}$ and coefficient of variation of 0.1386 , a value for $s_{i}$ of 0.3054 was obtained. Since the second estimate, $0.3054 \mu \mathrm{m}^{3}$, is less than the first, $0.3212 \mu \mathrm{m}^{3}$, we drew the conclusion that cells born without a split wall band are smaller on average than those with a growth zone.

The third approach, giving an estimate of size of nascent cells, $s_{i}$ (see section $\mathrm{V}$ of Table 1), depended on the volumes of cells having a birth site. This included cells whose wall bands had split both before and after division. The length of time it takes a cell to grow through the $0.06 \mu \mathrm{m}^{3}$ volume window is quite long: an $83 \mathrm{~min}$ cell grows from $0.3212 \mu \mathrm{m}^{3}$ to 
$0 \cdot 3212+0.06 \mu \mathrm{m}^{3}$ in $83 \log _{2}(0 \cdot 3812 / 0 \cdot 3212)=20 \cdot 5 \mathrm{~min}$, whereas the length of time it takes for a cell to grow from $s_{i}$ of 0.3212 to the average observed size of cells in this category of $0.3475 \mu \mathrm{m}^{3}$ is $9.4 \mathrm{~min}$. Therefore, about half of the cells observed with primary birth sites had split their wall band in the previous cell cycle, and half in the current cycle.

\section{Pole time}

The mean duration from the band splitting event until the corresponding fission event leads to completion of the resultant poles and to cell division was designated 'the mean pole time' and symbolized by $W$ (Shockman et al., 1974). In theory, it would be exactly equal to the doubling time if new growth zones were formed only at the instant of division. The mean pole time was expected to be much larger than the doubling time (i.e. $\bar{W}>T$ ), particularly for the more rapidly growing culture because of the high frequency of 'premature' wall band splitting. However, when the detailed calculation was done, it was found for both growth rates that the average pole time is not much greater than the mass doubling time.

The mean pole time was calculated in two independent ways. One approach was to calculate the average time that elapses between the event of secondary wall band splitting and the next cell division. The mean cell size at which secondary growth zones arose was calculated; this was done by subtracting one-half of the window volumes, i.e. $0.03 \mu \mathrm{m}^{3}$, from the mean size of cells with secondary birth sites. Then the time to grow from this size to the mean cell size for division, $e_{f}$, which occurs when that primary growth zone is completed, was calculated. To this, $T$ was added to get the mean pole time. These values were appropriately averaged with the $W$ values of the unit cells fraction of the population. This led to pole times of $0.31\left[83+83 \log _{2}[0 \cdot 6423 /(0 \cdot 5485-0.03)]+0.69[83-2]=89.6 \mathrm{~min}\right.$ for the $83 \mathrm{~min}$ culture and of $0.84\left[30+30 \log _{2}[0 \cdot 6789 /(0 \cdot 6010-0.03)]+0 \cdot 16[30-0 \cdot 1]=36 \cdot 3 \mathrm{~min}\right.$ for the 30 min culture.

These values are fully consistent with the second approach, the calculations for which are shown in Table 1. This approach depended on the mean sizes of $E$-phase cells with 0,1 or 2 growth zones. To carry out these calculations, the experimentally determined cell volumes in the sub-categories were used, corrected for the volume of secondary growth zones (if any). These volumes are designated as $e_{i_{0}}, e_{i_{1}}$, and $e_{i_{2}}$ in Table 1 . For each of the three categories of cells, the mean time from initiating wall band splitting until cell division can be calculated from these values, $e_{f}$, and $T$ using the formulae $\left[T \log _{2}\left(e_{f} \mid e_{i_{0}}\right), T \log _{2}\left(e_{f} \mid e_{i_{1}}\right)\right.$, and $\left.T \log _{2}\left(e_{f} \mid e_{i_{2}}\right)\right]$ for the populations with 0,1 or 2 growth zones. These times are only a few minutes except for those cells with two secondary growth zones. However, these times are overestimates because of statistical factors which must be considered when calculating the mean differences between the pole and mass doubling time. These statistical factors arise as follows: consider first the $E$-phase cells with no secondary growth zones. It is possible that both of the wall bands would have divided the next instant had we not added the fixative. At the other extreme, neither wall band might have split until division, or very shortly thereafter. A maximum estimate of pole time results from the assumption that band splitting would have happened in the next instant of time after the cells were fixed, i.e. $\left[T\left(1+\log _{2}\left(e_{f} f e_{i_{0}}\right)\right]\right.$, the minimum time is the mean doubling time, $T$. The average time lies somewhere in between, and we have taken this time to be the mean. That is the basis of the 0.5 in the first formula in section VII of Table 1 . When one zone is already formed, a statistical factor of 0.25 applies. No statistical factor applies if two secondary growth zones are observed. The weighted average was computed from these three times. Finally, a correction for the duration of unit cells was applied. This factor, as we have already noted, is due to some cells remaining as unit cells for a short time after division. The correction to the pole time is extremely small for the $30 \mathrm{~min}$ population because unit cells are so rare. For the 83 min population, we must take into account the proportion of cells that go through a unit cell stage: $69 \%$ of the nascent newborn cells arise as unit cells. Therefore, the correction amounts to approximately $2 \times 0.69=1.4 \mathrm{~min}$. This value was used to compute the final value given in Table 1 for the 83 min population. Evidently, neither type of correction alters the interesting conclusion that even in the fast growing cells, the mean pole time is not much greater than the mass doubling time. 


\section{DISCUSSION}

Because the cycle time of prokaryotes is highly variable, analysis of the duration of cell cycle phases from the frequencies of organisms in that phase observed in balanced growth is difficult. Clearly, application of equations based on the assumption of no cycle variability can only give tentative answers. The approach of Koch \& Higgins (1982) provides a reliable way when cell cycles are variable - but only under certain conditions. This is because the equations and graphs were made for a very specific model of the cell cycle: all cells were assumed to grow deterministically and exponentially, and to divide into two identical daughter cells when they achieved a size that has modest variation from cell cycle to cell cycle. It was further assumed that fluctuations from the mean division size would not correlate with fluctuations in the next cell cycle. These are quite good approximations for Escherichia coli (Koch, 1980), but not necessarily for other organisms. For $S$. faecalis, the size of the newly completed pole has some small variation, but we have no experimental information on the other items except for contradictory statements concerning the growth law for the cells. Mitchison (1961) observed a slowing in the increase of dry weight measured interferometrically toward the end of the cell cycle. However, the size of the organism is so small that this method was used at the experimental limit. Conversely, tracer data presented by Hinks et al. (1982b) on partially synchronized cells indicated exponential growth.

Fortunately, the method of Koch \& Higgins (1982) can itself provide a critical test of its own applicability. It was noted by Koch (1966) that the skewness of the size distribution was the most sensitive parameter reflecting the kinetics of cell growth. Figure $3(a)$ shows several hypothetical growth laws. They relate age $(a)$ and mass $(m)$ according to $a=-1+2 p+(1-3 p) m+p m^{2}$. Linear growth corresponds to $p=0$, exponential growth is approximated in the vicinity of $p$ $=-0 \cdot 2$ to $-0 \cdot 3$. Values of $p$ greater than 0 correspond to a slowing of growth toward the end of the cycle, such as takes place in most eukaryotic cells. Fig. 3(b) shows the canonical distributions and Fig. $3(\mathrm{c})$ the distributions when the critical size for division has a $10 \%$ coefficient of variation. It can be seen that the shape of the distributions is very sensitive to the value of $p$. Comparison of Fig. 3(b) with Figs $1(a)$ and $1(b)$ shows that the assumption of hypolinear $(p>0)$ growth and even linear growth should be rejected.

It might be objected that we have biased the conclusions by rejecting a few large cells from the distributions analysed. Even with these few cells added back to the distribution, hypolinear or linear growth would be rejected. Moreover, most of the rejected cells were so much larger than the rest that they could logically have been rejected because they appeared to be much larger than the largest cells forming part of the continuous distribution.

The data analysed here make it clear that the splitting of wall bands to create new growth zones occurs mainly close to the time when an old growth zone is almost completed and is functioning insufficiently fast to accommodate the production of new protoplasm. That is, we suggest that splitting occurs either shortly before division or immediately afterwards as a result of the inability of the functional growth zones of the cells to provide wall area fast enough to enclose newly created cytoplasm. If at the moment of fission (i.e. at the time when the old growth zone ceases to function) there is no growth zone, a growth zone arises in 2-3 min for cells with a mass doubling time of $83 \mathrm{~min}$ and much faster in the $30 \mathrm{~min}$ culture. We suggest that this rapid generation of a growth zone is necessary because the old wall is quite rigid and is already extended to its limit and can stretch little more. The pole volume does expand somewhat after completion of the septum. This can be seen from Fig. 7 of Higgins \& Shockman (1976), but the amount is not great $(30 \%)$. However, the pole volume probably changes little subsequent to completion of splitting of the septum. As pointed out previously (Koch, 1983), the hydrostatic pressure inside the cell wall can increase very rapidly when metabolic systems are normal, but wall enlargement is blocked. We suggest that this is the trigger that initiates wall band duplication.

For the rapidly growing cell population, where $84 \%$ or more of the cells at the instant they arise by cell division already have a growth zone, our corrected estimate of the pole time has an a verage value only a little larger $(6.7 \mathrm{~min})$ than the doubling time. In the actual population, the temporal relationship of a wall band splitting event and the next cell division event has a moder- 

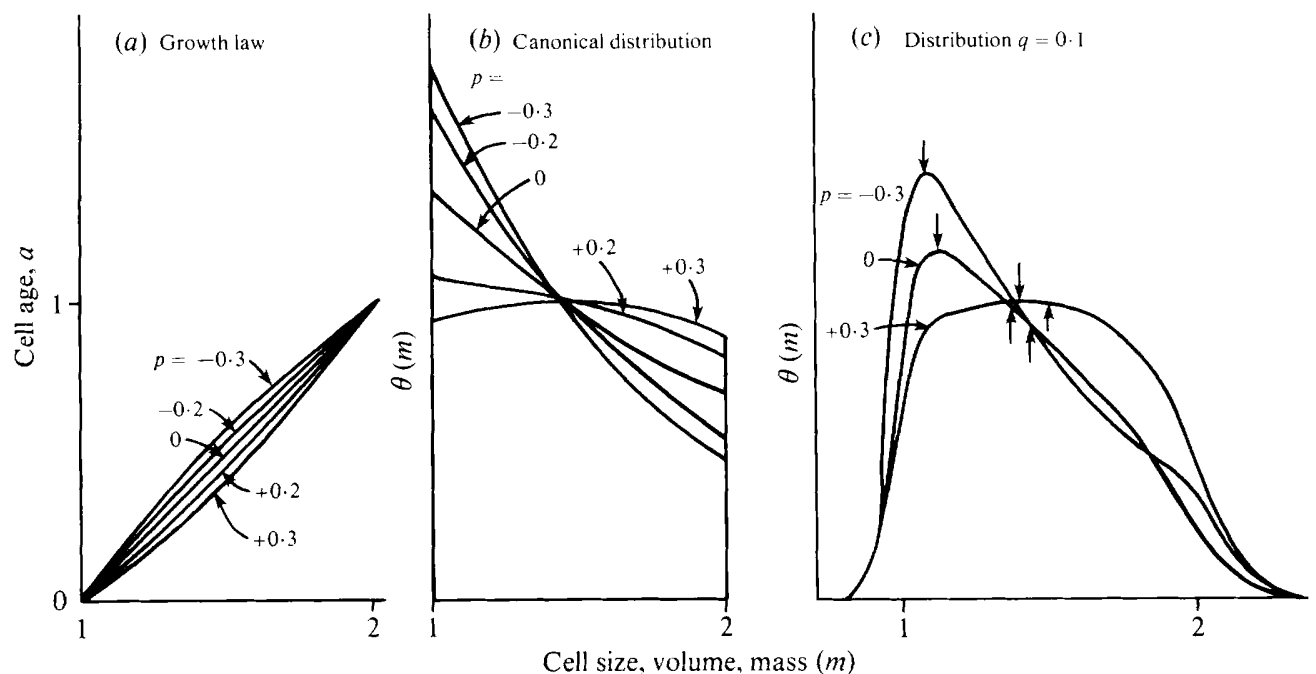

Fig. 3. Cell size distributions for different growth laws. See text. Arrows pointing downward indicate the mode, those pointing upward indicate the mean.
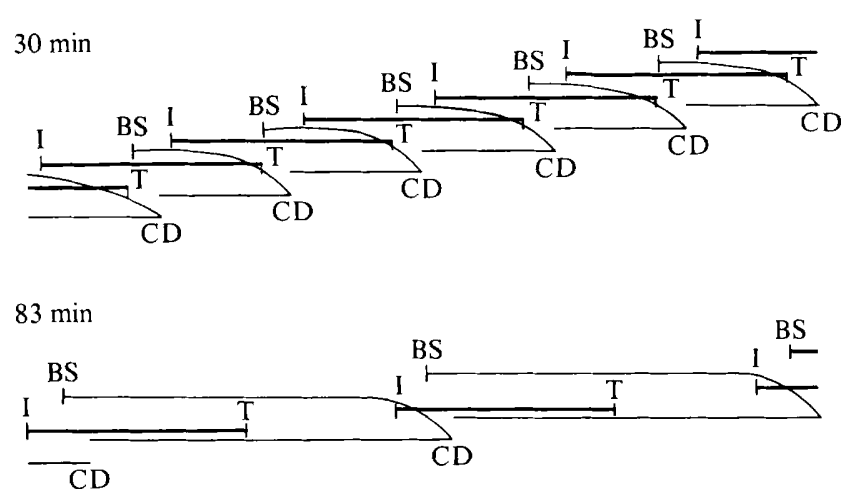

Fig. 4. Representation of the cell cycle at two growth rates. I, initiation of chromosome replication; T, termination of chromosome replication; BS, band splitting events; $C D$, cell division or fission events.

ate variability, since the size of cells with secondary birth site growth zones has a moderate variability (coefficient of variation of 0.22 and 0.15 for the $83 \mathrm{~min}$ and $30 \mathrm{~min}$ populations).

Since the $C$ time is nearly constant at 50-52 min (Hinks et al., 1978 b, Higgins et al., 1974), and much larger than the 30 min mass doubling time of the rapidly growing culture studied here, dichotomous replication must occur on nearly every replication cycle. Consequently, the initiation of chromosome replication does not occur synchronously with wall band splitting and the formation of growth zones. Rather, the rounds of replication must have been completed early enough so that segregation can take place (possibly the status of chromosome replication exercises a veto). Consequently, both cell division and chromosome replication are indirectly controlled by wall band splitting which in turn responds to the success of the cell in growing and producing new biomass (Koch \& Schaechter, 1962; Koch, 1971, 1977). Diagrams of the temporal relationships, using available estimates for $C$ and $D$ from the literature are shown in Fig. 4. Since the amount of wall in a completed pole is very nearly the same in cells growing at the two growth rates, a second important conclusion can be drawn. It is the converse of the first, and is that Gram-positive streptococci respond to alterations of nutritional conditions mainly by altering the rate at which they fashion the cell wall. Only to a small extent do they respond by shifting the timing of wall band splitting to earlier portions of the cycle. Contrariwise they alter 
the timing of initiation of chromosome replication in relation to the cell division cycle to adapt to different environmental conditions. A similar conclusion has been drawn by Gibson et al. (1983) by a different type of analysis of populations of replicas of $S$. faecalis cells. Their work considers a larger range of growth rates, but analyses only the relative frequencies of cells with different numbers of secondary growth zones, and the mean volume of cells with secondary birth sites, using equations based on the assumption that $T$ is the same for all cells in the culture. Their work and this paper complement each other because the present, more complete analysis, which utilizes several additional kinds of information, is less subject to approximations in fitting data to idealized models of the cell cycle. They in addition draw the conclusion that the wall band appears to split when the relevant portion of the cell approaches $0.261 \mu \mathrm{m}^{3}$ independently of the growth rate.

Dr Lolita Daneo-Moore is responsible for bringing the authors to work on this problem. She and Dr Carolyn Gibson criticized drafts of this paper severely as needed. Work in the authors' laboratories is supported by NSF 79 11241 (A.L.K.) and NIH Al-10971 (M.L.H.). A.L.K. was Guggenheim Fellow during a portion of this work.

\section{REFERENCES}

Edelstein, E. M., Rosenzweig, M. S., DaneoMOORE, L. \& Higgins, M. L. (1980). Unit cell hypothesis for Streptococcus faecalis. Journal of Bacteriology 143, 499-505.

Gibson, C. W., Daneo-Moore, L. \& Higgins, M. L. (1983). Initiation of wall assembly sites in Streptococcus faecalis. Journal of Bacteriology 154, 573579.

Higgins, M. L. \& Shockman, G. D. (1976). Study of a cycle of cell wall assembly in Streptococcus faecalis by three-dimensional reconstruction of thin sections of cells. Journal of Bacteriology 127, 1346-1358.

Higgins, M. L., MoOre, L. D., BoOthBY, D. \& Shockman, G. D. (1974). Effect of inhibition of deoxyribosenucleic acid synthesis on the direction of cell wall growth in Streptococcus faecalis (ATCC 9790). Journal of Bacteriology 118, 681-692.

Hinks, R. P., Daneo-Moore, L. \& Shockman, G. D. $(1978 a)$. Cellular autolytic activity in synchronized populations of Streptococcus faecium. Journal of Bacteriology 133, 822-829.

Hinks, R. P., Daneo-Moore, L. \& Shockman, G. D. $(1978 b)$. Approximation of the cell cycle in synchronized populations of Streptococcus faecium. Journal of Bacteriology 134, 1188-1191.

$\mathrm{KoCH}, \mathrm{A}$. L. (1966). Distribution of cell size in growing cultures and the applicability of the Collins-Richmond principle. Journal of General Microbiology 45, 409-417.

KoCH, A. L. (1971). The adaptive responses of
Escherichia coli to a feast and famine existence. Advances in Microbial Physiology 6, 147-217.

KocH, A. L. (1977). Does the initiation of chromosome replication regulate cell division? Advances in Microbial Physiology 16, 49-98.

KOCH, A. L. (1980). Does variability of the cell cycle result from one or many chance events? Nature, London 286, 80-82.

KoCH, A. L. (1983). The surface stress theory of microbial morphogenesis. Advances in Microbial Physiology 24, 301-366.

Koch, A. L. \& Blumberg, G. (1976). Distribution of bacteria in the velocity gradient centrifuge. Biophysical Journal 16, 389-405.

Koch, A. L. \& Higgins, M. L. (1982). Cell cycle dynamics inferred from the static properties of cells in balanced growth. Journal of General Microbiology 128, 2877-2892.

Koch, A. L. \& Schaechter, M. (1962). A model for statistics of the cell division process. Journal of General Microbiology 29, 435-454.

Mirchison, J. M. (1961). The growth of single cells: III. Streptococcus faecalis. Experimental Cell Research 22, 208-225.

Mitchison, J. M. (1971). The Biology of the Cell Cycle. London: Cambridge University Press.

Shockman, G. D., Daneo-Moore, L. \& Higgins, M. L. (1974). Problems of cell wall and membrane growth, enlargement, and division. Annals of the New York Academy of Sciences 235, 161-197. 